Int. J. Biol. Chem. Sci. 11(2): 744-756, April 2017

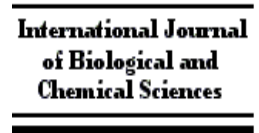

ISSN 1997-342X (Online), ISSN 1991-8631 (Print)

Original Paper http://ajol.info/index.php/ijbcs http://indexmedicus.afro.who.int

\title{
Les déterminants socioéconomiques de l'adoption des technologies améliorées de production du niébé diffusées par les champs écoles paysans dans les régions de Maradi et Zinder au Niger
}

\author{
Mahamane Moctar RABE ${ }^{1}$, Ibrahim BAOUA ${ }^{2 *}$, Razack ADEOTI ${ }^{3}$, Lawali SITOU ${ }^{2}$, \\ Laouali AMADOU ${ }^{1}$ et Barry PITTENDRIGH ${ }^{4}$ et Saadou MAHAMANE ${ }^{2}$ \\ ${ }^{1}$ Institut National de la Recherche Agronomique du Niger (INRAN) BP 240 Maradi Niger. \\ ${ }^{2}$ Université DanDicko DanKoulodo de Maradi, BP 465 Maradi, Niger. \\ ${ }^{3}$ Institut International d'Agriculture Tropicale (IITA), BP 0932 Cotonou, Bénin. \\ ${ }^{4}$ Michigan State University, Center for Integrated Plant System 578 Wilson Rd. Room 203 East Lansing, \\ MI 48824 USA. \\ *Corresponding author; E-mail: baoua.ibrahim@gmail.com
}

\section{REMERCIEMENTS}

Cette étude a été réalisée grâce au soutien financier et matériel du Programme de Productivité Agricole en Afrique de l'Ouest (PPAAO) dans le cadre du projet "Augmentation de la production du niébé par la mise en ouvre des champs écoles paysans" et du projet Leg-Lab Innovation financé par l'US Agency for International Development (USAID) et les opinions exprimées n'engage que les auteurs et ne reflète pas les points de vue du PPAAO et l'USAID.

\section{RESUME}

Le niébé est une denrée de base en Afrique subsaharienne. Le projet champ école paysan en a introduit des nouvelles technologies améliorées afin d'accroître son rendement dans les régions de Maradi et Zinder. Cette étude qui a concerné un échantillon de 300 producteurs a pour dessein d'identifier les contraintes entomologiques de la culture du niébé et de déterminer les facteurs affectant l'adoption des technologies agricoles. Le modèle de régression LOGIT a été utilisé comme outils d'analyse et les principaux résultats suivants ont été obtenus : 1) la punaise brune (Clavigralla tomensicollis Stall) et le puceron (Aphis craccivora Koch) sont les principaux insectes ravageurs de la culture pour 53 à $61 \%$ des répondants ; 2 ) les taux moyens d'adoption par technologie sont de 74,9\% pour les variétés améliorées, 57,2\% pour les pesticides chimiques, $20 \%$ pour la combinaison fumure organique/NPK ; $21.7 \%$ pour l'engrais NPK seul, $7,4 \%$ pour les biopesticides à base de graines de Neem et 5,7\% pour le respect de la densité de semis. Ces ratios sont 2 à 16 fois plus élevés chez les producteurs formés par rapport aux non formés ; 3) les variables «formations par les champs écoles paysans » et «l'accès au crédit» ont influencé positivement l'adoption des variétés améliorées, des biopesticides et des fertilisants ; 4) le genre a été positivement déterminant dans le respect de la densité de semis et l'utilisation des fertilisants ; 5) l'âge des répondants a eu une influence significative dans le respect des écartements entre les poquets. Ces données qui ont permis d'apprécier les niveaux d'utilisation des technologies de développement de la culture du niébé pourraient aussi contribuer à améliorer les programmes de vulgarisation des technologies agricoles en zone rurale.

(C) 2017 International Formulae Group. All rights reserved.

Mots clés : adoption, technologies améliorées, niébé, champ écoles paysans, Maradi, Zinder. 


\title{
Socio-economic determinants for adoption of improved technologies disseminated through Farmer Field Schools for cowpea production in the regions of Maradi and Zinder in Niger
}

\begin{abstract}
Cowpea is a staple food in sub-Saharan Africa. The Farmer Field School project introduced new improved technologies to increase cowpea's yield in Maradi and Zinder regions. This study, involved 300 producers, its aim is to identify the entomological constraints of cowpea production and determine the factors affecting the adoption of agricultural technologies. The LOGIT regression model was used as an analytical tool and the following main results were obtained: 1) pod sucking bug Clavigralla tomensicollis Stall and Aphids, Aphis craccivora Koch are the main insect pests of the crop according 53 to $61 \%$ of respondents; 2) the average adoption rates per technology were $74.9 \%$ for improved varieties, $57.2 \%$ for chemical pesticides, $20 \%$ for organic fertilizer and NPK combination; $21.7 \%$ for the NPK fertilizer, $7.4 \%$ for Neem seeds' biopesticides and $5.7 \%$ for the respect of sowing density. These ratios were 2 to 16 times higher with trained producers compared with untrained ones; 3 ) the variables "training by farmer field school" and "access to loan" have positively influenced the adoption of improved varieties, biopesticides and fertilizers; 4). The gender was positively determinant for application of recommended sowing density and the use of fertilizers. 5) Age of respondents had significant difference in term of adopting sowing density. These data, helped to assess the practice levels of technologies related to cowpea production and could also help to improve rural extension programs about agricultural technologies.
\end{abstract}

(C) 2017 International Formulae Group. All rights reserved.

Keywords: adoption, improved technologies, cowpea, Farmers Field Schools. Maradi, Zinder.

\section{INTRODUCTION}

Le Niger, avec un système de production agricole diversifié, est dominé par la culture du mil pour les céréales, du niébé et de l'arachide pour les légumineuses. Cette production agricole se pratique sur des petites exploitations familiales cultivées en système pluvial (RECA, 2008).

Le niébé est cultivé dans toute la bande agricole Sud du Niger. Dans les régions de Maradi et Zinder, il occupe $48 \%$ des superficies emblavées et représente le troisième produit agricole contributeur au PIB après le bétail et l'oignon (MA, 2012). Au cours des dix dernières années, plusieurs nouvelles variétés de niébé ont été proposées aux producteurs par les structures de recherche nationales et régionales. Seize variétés figurent dans le catalogue national publié en 2012 (RECA, 2012)

Des technologies relatives à la gestion de la fertilité des sols et des ravageurs ont été mises au point (INRAN, 2013). Malheureusement, les rendements de la culture demeurent faibles variant entre 297 à
$332 \mathrm{Kg} / \mathrm{ha}$ (MA, 2015). Plusieurs contraintes ont été évoquées pour justifier cette situation telles que le faible encadrement agricole des producteurs avec un taux d'un agent pour 35 villages (MA, 2012); la pression des ravageurs des cultures: les punaises, les pucerons et les chenilles occasionnent des pertes importantes de rendement (Egho, 2011); Le coût élevé des pesticides de bonne qualité et les méthodes alternatives non chimiques très peu vulgarisées; une faible adoption des génotypes de niébé performants (Ibro et Bokar, 2001).

La diffusion de ces technologies améliorées est effectuée par les services de vulgarisation qui utilisent des outils tels que la fiche technique et le champ expérimental, les messages. Le mécanisme traditionnel topdown suit un schéma linéaire où le producteur recevait les recommandations de pratiques pas toujours élaborées avec sa participation. La démarche participative consiste à identifier d'abord la demande des producteurs. Le mécanisme est plus ou moins participatif en 
fonction des pays et les approches utilisées sont variables d'un pays à l'autre.

Au Niger, le processus « champ école paysan » (CEP) a débuté en 1999 avec le projet PRONAF (Projet Niébé Afrique) de IITA. Au cours des dix dernières années, plus de 2000 CEP ont été mis en œuvre avec les projets de développement. La FAO à travers les projets Intrants et IARBIC a animé 364 CEP qui ont concerné surtout le mil et le sorgho dans toutes les régions agricoles du pays entre 2008 et 2011. Une hausse de rendement de $366 \%$ pour le niébé, $200 \%$ pour l'arachide, $169 \%$ pour le mil et $100 \%$ pour le sorgho grâce l'intégration de nouvelles technologies est obtenu (FAO, 2011). L'approche CEP a été considérée plus efficace que la démonstration ou le «training and visit» parce qu'elle permet une plus grande implication et responsabilisation des producteurs (Okoth et al., 2006).

L'objectif principal de cette étude est d'identifier les principaux ravageurs des cultures et d'analyser les déterminants de l'adoption des technologies améliorées du niébé dans les zones couvertes par les CEP sur l'adoption de ces technologies.

\section{MATERIEL ET METHODES \\ Zone d'étude}

La présente étude a été conduite du 28/07/2015 au 30/08/2015 dans les régions de Maradi et Zinder localisées dans la partie centre-sud agricole du Niger. Elles ont une pluviométrie moyenne variant entre 300 et $600 \mathrm{~mm}$ et des pratiques culturales similaires (mil, sorgho, niébé, arachide). La densité de la population est de 100 habitants $/ \mathrm{km}^{2}$ (MA, 2012). Ces régions ont été concernées par l'implantation du processus CEP au cours de ces cinq dernières années.

\section{Questionnaire}

Les données ont été recueillies au moyen d'un entretien individuel à l'aide d'un questionnaire structuré. La fiche d'enquête comprenait plusieurs parties: 1) les caractéristiques socio-économiques des répondants, 2) les contraintes de la production du niébé, 3) les technologies diffusées ; 4) le nombre de producteurs qui utilisent et continuent à les utiliser les technologies diffusées.

\section{Échantillonnage}

Un échantillonnage raisonné de 300 chefs d'exploitations dont 150 à Maradi et 150 à Zinder ou unité de production a été effectué dans 10 villages de la zone d'étude, soit 30 producteurs par village. Dans le choix des villages, il a été priorisé les zones d'intervention des fédérations ayant conduit au moins une fois le champ école paysan. Les villages retenus dans l'échantillon sont les sites expérimentaux des projets de développements, DGP/CRSP et Legume Lab Innovation de I'INRAN, PASADEM du FIDA, Intrants et IARBIC de la FAO et CEP du PPAAO ayant intervenu entre 2008 et 2015 dans la région de Maradi. La région de Zinder a été concernée uniquement par les deux derniers programmes.

Les producteurs formés dans les CEP ont été sélectionnés à partir des listes fournies par les organisations paysannes ayant participé à la mise en œuvre du processus au niveau des villages ciblés. L'échantillon a été complété par les producteurs des villages n'ayant pas été impliqués dans le CEP.

\section{Analyse des données}

Dans la littérature, il existe plusieurs modèles d'analyse pour l'estimation des taux d'adoption des technologies et de leurs facteurs déterminants.

\section{Les modèles dichotomiques simples}

Ces modèles expliquent des phénomènes dont les manifestations ne peuvent prendre que deux valeurs 0 et 1 . Dans la littérature, trois types de modèles sont principalement utilisés pour analyser la décision des producteurs à adopter une technologie agricole: les modèles de probabilité linéaire, de LOGIT et de PROBIT. Le premier modèle présente des inconvénients parce que la probabilité peut souvent dépasser 1. Les deux derniers modèles sont les plus couramment utilisés pour spécifier les 
relations entre la probabilité de choix et les variables déterminantes du choix (CIMMYT, 1993). Le LOGIT a l'avantage de faciliter l'interprétation des paramètres $\beta$ associées aux variables explicatifs xi (Amemiya, 1981). La fonction de répartition $\mathrm{F}($.) correspond à la fonction logistique $\forall \mathrm{w} \in \mathrm{R}$ :

$$
F(w)=\frac{e^{w}}{1+e^{w}}=\frac{1}{1+e^{-w}} \Delta(w)
$$

Ce modèle définit la probabilité associée à l'événement yi $=1$, comme la valeur de la fonction de répartition de la loi logistique considérée au point xi $\beta$ :

$$
P i=\Delta\left(x_{i} \beta\right)=\frac{1}{1+e^{-\left(\beta 0+\beta i^{Z}\right)}} \forall i=1 \ldots . N
$$

Avec:

$W=\beta 0+\beta 1$ ZONEAGR +

$\beta 2 Z O N E P R O X+\beta 3 P R O X M A R C H+\beta 4$ SEXE

$+\beta 5 A G E+\beta 6 E D U C A T+\beta 7$ ACTIFAGRI

$+\beta 8$ OP $+\beta 9$ ACCREDIT $+\beta 10$ CONTACEP

$+\beta 11$ SUCFACAGRI

Où $\beta 0$ est le terme constant ; $\beta \mathrm{i}$ les

coefficients à estimer.

Le logiciel SPSS a été utilisé pour les statistiques descriptives et les régressions économétriques.

\section{RESULTATS}

\section{Caractéristiques des producteurs enquêtés}

A partir de l'analyse des résultats de l'étude, la structure de l'échantillon par sexe montre que $38.3 \%$ des producteurs du niébé sont des femmes. $14.8 \%$ de ces femmes $(25 \%$ des répondantes de la région de Maradi et 4,6\% de celles de la région de Zinder) ont pris part activement au processus CEP. L'âge moyen des producteurs est de $46 \pm 20$ ans pour les femmes et $45 \pm 18$ ans pour les hommes soit une moyenne de $45.31 \pm 17$ ans pour les deux sexes. Les enquêtés sont en majorités mariés $(92,3 \%)$. Il ressort aussi de cette analyse que le niveau d'instruction reste relativement faible avec seulement $32 \%$ qui ont fréquenté l'école primaire, $5,3 \%$ le niveau secondaire et $47,3 \%$ ne sont ni instruits ni alphabétisés. Cependant, $52,7 \%$ affirment avoir été formés sur le processus champ école paysan. L'agriculture est la principale activité pour $99,3 \%$ des producteurs.

Pour le crédit, $17 \%$ des producteurs ont affirmé avoir accès: $30,7 \%$ à Maradi et 3,3\% à Zinder $(\chi 2(2$ d.f $)=39,7 ; \mathrm{p}<0,001) ; 26.6 \%$ pour les formés et $6.3 \%$ pour les non formés $(\chi 2(2 \mathrm{~d} . \mathrm{f})=21,72 ; \mathrm{p}<0,001)$;

Le taux moyen d'adhésion aux Organisations des producteurs est de $58,3 \%$ dont $72,7 \%$ à Maradi et $44,0 \%$ à Zinder $(\chi 2(2$ d.f $)=25,35 ; \mathrm{p}<0,001) ; 94,3 \%$ pour les formés et $18.3 \%$ pour les non formés $(\chi 2(2 d . f)=177,69 ; p<0,001)$.

\section{Les principales contraintes entomologiques du niébé}

Dans les deux régions, la punaise brune Clavigralla tomensicolis Stall a été le ravageur le plus mentionné par les répondants suivie du Puceron du niébé Aphis craccivora (Tableau 1). La punaise brune est observée pendant la période de fructification. Elle suce les gousses et provoque l'avortement des graines causant souvent une perte totale des récoltes. Le puceron attaque la plante de la levée à la maturité. Il cause le rabougrissement et est responsable de la faible production des fleurs et des gousses.

L'incidence des Mylabres (Mylabris sp.) et du criquet sénégalais Oedaleus senegalensis Krauss a été mentionnée par une proportion importante des répondants de la région de Zinder. Mylabris sp. détruit les fleurs et les jeunes gousses. Le criquet sénégalais est responsable de la défoliation des plants de niébé en début et à la fin de saison pluvieuse.

\section{Inventaire des technologies introduites à travers l'approche CEP}

Il a été identifié huit (8) variétés améliorées provenant des instituts de recherche comme l'Institut international d'Agriculture Tropicale (IITA) : IT90k 372-1-2, IT99K 5731-1, IT89KD 374-57, IT97K449-35, IT98 K 205-8; INRAN : TN 5-78, IT97 K 499-38, et l'INERA : KVX 30-309-6G, qui ont différentes caractéristiques (cycle, la couleur et la taille des graines).

Pour la protection des cultures, il a été 
inventorié deux technologies à savoir : L'utilisation des extraits aqueux de graines de Neem (Azadirachta indica Juss) avec la préparation d'une solution avec $500 \mathrm{~g}$ de poudre de graines pour 10 litres d'eau et la pulvérisation de 250 litres de la solution à l'hectare et la lutte chimique avec le Diméthoate $50 \mathrm{EC}$ à la dose de 1litre/ha.

Pour le redressement de la fertilité des sols, il a été recensé l'usage de l'engrais minéral 15-15-15 en micro dose (NPK) et l'application de la fumure organique combinée à la fumure minérale $(\mathrm{FO}+\mathrm{NPK})$.

\section{Les taux d'adoption des technologies diffusées à travers les CEP}

Selon les régions, les taux d'adoption des technologies individuelles ont varié de 0.7 à $80.0 \%$ (Tableau 2). Les taux les plus élevés concernent les variétés améliorées, l'utilisation des pesticides et de l'engrais composé NPK. Les ratios d'adoption pour 8 des 9 technologies recensées sont 1.1 à 61 fois plus importants dans la région de Maradi comparée à celle de Zinder. Les écarts entre les taux d'adoption des deux régions sont de $42,0 \%$ pour l'utilisation du NPK, 40,0\% pour l'application de la combinaison $\mathrm{FO}+\mathrm{NPK}, 20,6 \%$ pour le respect de la densité de 3 plants par poquet, 14,0\% pour l'utilisation des graines de Neem dans la lutte contre les insectes ravageurs du niébé, $10,0 \%$ pour toutes les variétés améliorées.

Dans le cas des variétés, les génotypes IT 90K 372-1-2 et IT 99K 573-1-1 présentent les taux d'adoption les plus élevés dans la région de Maradi avec respectivement des écarts de 17,0 et $17,3 \%$ par rapport à la région de Zinder. La variété KVX 30309 6G a présenté un taux d'adoption plus élevé dans la région de Zinder avec un écart de 10,7\% par rapport à la région de Maradi.

En considérant le facteur «formation par le processus CEP», les taux d'adoption calculés par technologie ont varié de 7,6 à $85,5 \%$ pour les producteurs formés et entre 0,8 et $67,3 \%$ pour les non formés. Les ratios sont 1,36 à 16,25 fois plus élevés pour 7 des 9 technologies recensées dans le cas des producteurs formés par rapport à ceux non formés. Les écarts entre les deux groupes sont de $22,6 \%$ pour toutes les variétés améliorées, $12,3 \%$ pour l'utilisation du génotype IT $90 \mathrm{~K}$ 373-1-2 et 9,6\% pour la variété IT 99K 573-1$1,26,5 \%$ pour l'application du NPK et $26 \%$ pour l'application de la combinaison FO+NPK ; 19,4 pour le respect de 3 plants par poquet, $10,8 \%$ pour le respect densité optimale de semis pour chacune de variétés et $12,2 \%$ pour l'utilisation du jus de graines de Neem dans la lutte contre les ravageurs. Le taux d'adoption entre les formés et le non formés sont comparables entre les deux régions pour l'utilisation des pesticides et du génotype KVX $303096 \mathrm{G}$.

\section{Facteurs déterminant l'adoption des technologies}

Sur les 12 facteurs étudiés avec le modèle LOGIT, quatre ont donné des coefficients de corrélations positifs et significatifs avec les technologies diffusées (Tableau 4). L'appartenance à un champ école paysan (CONTACEP) a été positivement corrélée aux taux d'adoption des technologies suivantes par ordre d'importance: 1) l'utilisation des variétés améliorées, 2) le respect de la densité des plants par poquet, 3) L'application de l'engrais composé NPK, 4) l'utilisation des extrait aqueux des graines de Neem et 5) l'application de la combinaison FO+ NPK. L'accès au crédit (ACCREDIT) a donné une corrélation positive avec l'utilisation du NPK et de la combinaison FO+NPK; la variable sexe (SEXE) a aussi influencé positivement les taux d'adoption de 4 technologies : 1) le respect des écartements entre les poquets de niébé ; 2) L'utilisation de l'engrais composé NPK ; 3) l'application de la combinaison $\mathrm{FO}+\mathrm{NPK}$ et 4) le respect de la densité de 3 plants par poquet. Les taux d'adoption de ces techniques sont plus élevés chez les hommes par rapport aux femmes; l'âge (AGE) a influencé significativement l'adoption de l'écartement entre les poquets. La technologie est plus utilisée par les producteurs âgés de moins de 45 ans. 
Tableau 1: Proportion des réponses des enquêtés par rapport aux principaux insectes nuisibles de la culture du niébé dans les régions de Maradi et Zinder.

\begin{tabular}{lccccc}
\hline & \multicolumn{3}{c}{ Proportion des répondants } & & \multicolumn{2}{c}{ Seuil de } \\
\cline { 2 - 4 } Ravageurs des cultures & Maradi & Zinder & \% Moyen & $\chi^{\mathbf{2}}$ (2d.f) & signification \\
\hline Puceron (Aphis craccivora Koch) & 52,0 & 54,7 & 53,3 & 0,214 & $\mathrm{~ns}$ \\
Punaise (Clavigralla tomensicollis Stall) & 66,0 & 56,7 & 61,3 & 2,75 & $\mathrm{~ns}$ \\
Mylabre (Mylabris sp.) & 33,3 & 46,7 & 40,0 & 5,556 & $* *$ \\
Criquet (Oedaleus senegalensis Krauss) & 10,7 & 59,3 & 35,0 & 78,08 & $* * *$ \\
\hline
\end{tabular}

${ }^{* *} \mathrm{p}<0,05 \quad{ }^{* * *} \mathrm{p}<0,001 \quad$ ns: différence non significative

Tableau 2 : Taux d'adoption des technologies améliorées de production du niébé dans les régions de Maradi et Zinder.

\begin{tabular}{|c|c|c|c|c|c|}
\hline & \multicolumn{3}{|c|}{ Proportion des répondants } & \multirow[b]{2}{*}{$\chi^{2}(2 d . f)$} & \multirow{2}{*}{$\begin{array}{c}\text { Seuil de } \\
\text { signification }\end{array}$} \\
\hline & Maradi & Zinder & Moyenne & & \\
\hline Respect de trois plants par poquet & 23,3 & 2,7 & 13,0 & 36,10 & $* * *$ \\
\hline Respect écartements entre les poquets & 10,7 & 0,7 & 5,7 & 14,03 & $* * *$ \\
\hline Apport NPK & 42,7 & 0,7 & 21,7 & 75,49 & $* * *$ \\
\hline Apport Combinaison FO+NPK & 40 & 0,0 & 20 & 75 & $* * *$ \\
\hline Utilisation pesticide chimique & 74,7 & 37,8 & 57,2 & 39,49 & $* * *$ \\
\hline Utilisation des extraits de graines de Neem & 14,0 & 0,0 & 7,4 & 20,40 & $* * *$ \\
\hline Variétés améliorées & 80,0 & 69,8 & 74,9 & 4 & $* *$ \\
\hline IT90k 372-1-2 & 70,0 & 53,0 & 61,5 & 9,10 & $* *$ \\
\hline IT99K 573-1-1 & 19,3 & 2,0 & 10,7 & 23,64 & $* * *$ \\
\hline KVX 30-309-6G & 1,3 & 12,0 & 6,7 & 13,71 & $* * *$ \\
\hline
\end{tabular}

Tableau 3: Taux d'adoption des technologies améliorées de production du niébé pour les producteurs formés et non formés à travers le processus CEP.

\begin{tabular}{lccccc}
\hline & \multicolumn{3}{c}{ Proportion des répondants } & & \multicolumn{2}{c}{$\begin{array}{c}\text { Seuil de } \\
\text { signification }\end{array}$} \\
\cline { 2 - 5 } & Non formés & Formés & Moyenne & $\chi^{\mathbf{2}}$ (2d.f) & sonct \\
\hline Respect de trois plants par poquet & 2,8 & 22,2 & 13,0 & 24,72 & $* * *$ \\
Respect écartements entre les poquets & 0,0 & 10,8 & 5,7 & 16,19 & $* * *$ \\
Apport NPK & 7,7 & 34,2 & 21,7 & 30,78 & $* * *$ \\
Apport Combinaison FO+NPK & 6,3 & 32,3 & 20 & 31,45 & $* * *$ \\
Utilisation pesticide chimique & 62,6 & 52,6 & 57,2 & 2,89 & $\mathrm{~ns}$ \\
Utilisation des extraits de graines de Neem & 0,8 & 13,0 & 7,4 & 15,49 & $* * *$ \\
Utilisation variétés améliorées & 62,9 & 85,5 & 74,9 & 20,37 & $* * *$ \\
IT90k 372-1-2 & 55 & 67,3 & 61,5 & 4,75 & $* *$ \\
IT99K 573-1-1 & 5,6 & 15,2 & 10,7 & 7,16 & $* *$ \\
KVX 30-309-6G & 5,6 & 7,6 & 6,7 & 0,4 & $\mathrm{~ns}$ \\
\hline
\end{tabular}


Tableau 4 : Résultat du modèle d'estimation des facteurs d'adoption des technologies améliorées diffusées.

\begin{tabular}{|c|c|c|c|c|c|c|}
\hline & Variétés & FO+NPK & NPK & $\begin{array}{c}\text { Extraits } \\
\text { aqueux des } \\
\text { graines de } \\
\text { Neem }\end{array}$ & $\begin{array}{c}\text { Respect des } \\
\text { écartements } \\
\text { entre les } \\
\text { poquets }\end{array}$ & $\begin{array}{c}\text { Respect de } 3 \\
\text { plants/poquet }\end{array}$ \\
\hline ZONEAGR & $-0,701$ & 21,966 & 21,383 & 19,937 & 18,819 & 19,586 \\
\hline ZONEPROX & $-0,457$ & $-0,169$ & 0,032 & $-0,362$ & $-0,294$ & 0,237 \\
\hline PROXIMARCH & 0,974 & 0,010 & $-16,700$ & $-0,315$ & 0,183 & $-18,010$ \\
\hline SEXE & $-0,485$ & $1,709 * *$ & $1,848 * *$ & 0,348 & $2,942 * *$ & $1,193^{* *}$ \\
\hline AGE & 0,039 & 0,030 & 0,028 & 0,000 & $0,077^{*}$ & $-0,009$ \\
\hline ANSEXPAG & $-0,052$ & $-0,025$ & $-0,028$ & $-0,035$ & $-0,052$ & $-0,004$ \\
\hline EDUCAT & $-0,410$ & 0,107 & $-0,051$ & $-0,663$ & $-0,558$ & 0,588 \\
\hline ACTIFAGRI & 0,093 & $-0,071$ & $-0,074$ & $-0,178$ & $-0,082$ & 0,005 \\
\hline $\mathrm{OP}$ & 0,506 & 0,619 & 0,334 & 1,389 & 1,580 & 0,869 \\
\hline ACCREDIT & 0,394 & $0,738^{*}$ & $1,003 * *$ & $-0,082$ & $-0,747$ & $-0,706$ \\
\hline CONTACEP & $3,149 * * *$ & $1,799 * *$ & $1,921^{* *}$ & $1,820^{* *}$ & 0,068 & $2,406^{* *}$ \\
\hline SUCFCAGRI & 0,053 & 0,190 & 0,101 & 0,455 & $-0,052$ & 0,641 \\
\hline Constant & $-0,305$ & $-25,905$ & $-8,287$ & $-21,913$ & $-25,501$ & $-6,137$ \\
\hline
\end{tabular}

\section{DISCUSSION}

Les résultats de cette étude donnent des informations nouvelles sur le processus CEP qui est un concept implanté au Niger depuis 17 années. Sa mise en œuvre a permis l'adoption de plusieurs technologies dans les régions de Maradi et Zinder.

Dans le cas des variétés améliorées, il a été identifié 8 variétés diffusées à travers les CEP et seulement trois de ces génotypes ont donné des taux d'adoption moyens variant entre 6.7 et $61,5 \%$. La variété IT 90K 372-1-2 est la plus utilisée avec un taux d'adoption de $55 \%$ chez les producteurs non formés et un taux plus important de $67,3 \%$ dans le groupe des producteurs ayant participé aux CEP. Ce taux est largement en deçà de celui rapporté par Ibro et Bokar (2001). Cette performance peut aussi s'expliquer par le potentiel de rendement de cette variété, sa précocité et sa résistance aux ravageurs (Dugje et al., 2009 ; Saidou et al., 2011). Cela confirme aussi les affirmations de (Bellon et al., 2006) qui ont stipulé que les producteurs adoptent les variétés améliorées afin de maximiser les avantages des caractéristiques tels que le rendement, la résistance aux maladies. En plus de l'IT90K 372-1-2, deux autres variétés l'IT 99K 573-1-1 et la KVX 30309 6G ont donné des taux d'adoption non négligeables variant entre 6.7 et $10.7 \%$. Cinq autres variétés, malgré qu'elles soient aussi précoces, productives et à résistance multiple sont faiblement utilisées par les producteurs. Cette situation s'explique certainement par la disponibilité de leurs semences en milieu rural. Les 3 génotypes qui connaissent un début d'adoption constituent $78.04 \%$ des 1854 tonnes de semences R1 et R2 répertoriées au niveau du catalogue national des semences améliorées après la campagne agricole 2015 (MA, 2016). Pour encourager l'utilisation des variétés améliorées, il serait important de développer des filières de commercialisation des produits agricoles qui les valorisent davantage. En Zambie, il a été démontré que les formations des producteurs, l'accès aux services de vulgarisation, la 
diffusion des informations sur le marché font parties des principaux facteurs déterminants de l'utilisation de semences améliorées (Khonje et al., 2014).

Les technologies de redressement de la fertilité des sols ont donné des taux d'adoption assez élevés. L'apport du NPK seul ou associé à la matière organique est pratiqué en moyenne par 20 à $21.7 \%$ des producteurs des deux régions. Le taux d'adoption de ces technologies est 4 à 5 fois plus élevé dans le groupe des producteurs formés. En effet, la pauvreté des sols constitue une contrainte permanente en milieu paysan sahélien (Osbahr, et Allan, 2003 ; Dutordoir, 2006). Les sols sableux sont carencés en phosphore et en azote (Bationo et al., 1989). L'apport de l'engrais composé NPK seul ou combiné à la matière organique permet d'augmenter la disponibilité en nutriments du sol et donc influencer positivement le rendement des cultures (Bationo et Mokwunye, 1991). Ces pratiques font bien partie des programmes développés dans les CEP et cela explique les taux d'adoption plus élevés au niveau des producteurs adhérents aux processus du CEP.

L'adoption des fertilisants est significativement affectée par le genre, l'accès au crédit et la formation CEP. Ceci confirme l'étude de (Dey, 1981) qui a démontré que les hommes ont plus accès à l'information et aux intrants par rapport aux femmes. Pour le crédit financier, il est souvent peu accessible aux femmes en raison des préjugés culturels, $\mathrm{du}$ fait des obstacles juridiques formels les empêchant de conclure des contrats en leur propre nom et aussi avec la méconnaissance des textes et conditions régissant le crédit rural. Elles ne possèdent souvent pas les éléments de patrimoine requis comme garanties par les institutions financières (Miller et al., 2009). Il faut aussi admettre qu'au Niger, les institutions de micro finance (IMF) sont encore très réticentes à investir dans le crédit rural du fait de l'accroissement des coûts opérationnels et du risque très élevé lié à l'agriculture. Ces IMF sont encore retranchées en milieu urbain, périurbain et rurbain pour diminuer leurs coûts et leurs risques (Hamadziripi, 2008). En plus, les taux d'intérêt pratiqués par les IMF sont supérieurs à $12 \%$ parce que fortement dépendants du taux d'intérêt de la Banque Centrale qui est de $6 \% / a n$. Les intérêts demandés ne sont pas à la portée des femmes qui font partie de la tranche la plus pauvre.

En dépit de tout cela, il faut admettre que la disponibilité et l'accès aux ressources financières peut renforcer l'adoption des technologies de gestion de la fertilité des sols. Selon Sale et al. (2014), l'accès au crédit est déterminant pour le choix d'une stratégie, car, les producteurs ayant un accès facile aux crédits auront tendance à mieux adopter les nouvelles technologies.

Pour la protection des cultures, il est noté un taux d'adoption des pesticides chimiques comparable entre les adhérents et les non adhérents au CEP. Il est bien connu que le niébé est bien adapté aux sols sahéliens, mais son rendement est conditionné par une bonne gestion des ravageurs (Oyewale et al., 2014). Les données de la présente étude confirment cette menace des ravageurs, car plus de $60 \%$ des producteurs ont mentionné la Punaise brune du niébé et plus de $50 \%$ ont fait cas des pertes liées aux pucerons. Dans la région de Zinder, d'autres ravageurs tels que les sautériaux et les mylabres ont été ajoutés à la liste des déprédateurs. Cette situation explique peut-être pourquoi plus de la moitié des producteurs des deux régions ont recours à la lutte chimique pour la protection du niébé.

Le taux moyen d'adoption des extraits botaniques a été 16 fois plus élevé chez les producteurs participants aux CEP. Ceux-ci semblent plus informés sur la technologie par rapport aux autres producteurs. L'efficacité des extraits botaniques dans la gestion des ravageurs du niébé est bien documentée 
(Jackai et Oyediran, 1991 ; Jackai et al., 1992) et aussi l'influence positive de l'approche CEP sur l'utilisation des méthodes alternatives non chimiques a été déjà démontrée par Djinadou et al. (2008).

Le taux moyen d'adoption des biopesticides à base de graines de Neem a été estimé à $7,4 \%$ pour les deux groupes des producteurs. Il reste comparable aux taux de 8\% présenté par Adéoti et al. (2002) au Niger. Donc, malgré les démonstrations effectuées dans les CEP, après 13 années, ce taux d'adoption est resté inchangé. Adéoti et al. (2002) a déjà évoqué la pénibilité liée à l'utilisation de cette technologie. Le traitement d'un hectare requiert 250 litres de préparation à base de Neem. Les superficies emblavées avec le niébé varient en moyenne entre 3,18 et 1,25 (MA, 2008). L'application de 2 à 4 traitements au biopesticide semble effectivement assez fastidieux.

Cependant, dans la présente étude, la variable superficie des champs (SUCFCAGRI) de niébé n'a pas influencé négativement l'utilisation des extraits du jus de grains de Neem. Selon les producteurs, la facilité d'accès et d'utilisation des pesticides chimiques explique le faible taux d'adoption de la technologie alternative non chimique (Djinadou et al., 2008)

Le nombre de plants de niébé et les écartements entre les poquets déterminent le nombre de plant de niébé par hectare. Les producteurs en utilisant ces technologies arrivent à obtenir la densité optimale et le rendement potentiel des variétés. Ces informations sont en général consignées dans les fiches techniques et les catalogues des espèces végétales. Le taux d'adoption de ces technologies est plus important dans le groupe des producteurs formés certainement en raison des séances pratiques effectuées lors de l'installation des CEP.

La variable AGE a influencé les taux d'adoptions relatifs à la densité de semis.
Cette technologie semble pénible pour les producteurs âgés en raison du temps de travail supplémentaire qu'occasionne l'utilisation du rayonneur.

Le niveau d'instruction des producteurs a toujours été présenté comme un facteur assez déterminant dans la diffusion des technologies agricoles (Nkamleu, 2000 ; Adesina, 2000). Dans cette étude, la variable niveau d'instruction (EDUCAT) n'a pas influencé l'adoption des technologies améliorées. Le processus CEP semble donc bien adapté pour l'encadrement des producteurs analphabètes. L'apprentissage des adhérents aux CEP est certainement facilité par les séances pratiques. Selon Schultz (1964), le fait d'amener le producteur à comprendre l'itinéraire technique des variétés améliorées lors des différentes formations illustratives dans le champ école, détermine significativement et influence positivement leur décision d'adoption.

L'adhésion aux OP n'a pas aussi influencé l'adoption des technologies. Tout de même, la mise en place de ces structures est à conseiller pour renforcer le niveau d'organisation et la cohésion entre les groupes des producteurs. Au Kenya et en Éthiopie, il a été démontré que l'adhésion des producteurs aux OP influence positivement sur l'adoption des fertilisants minéraux et des semences améliorées (Fischer et Qaim, 2012; Abebaw et Haile, 2013).

Les taux d'adoption des technologies ont été plus élevés dans la région de Maradi par rapport à celle de Zinder. Les écarts les plus importants concernent l'utilisation des fertilisants et des pesticides et peuvent être expliqués par le faible taux d'encadrement de la région de Zinder (MA, 2012) et le nombre réduit des projets ayant implanté le processus CEP au cours de ces dernières années dans la région. 


\section{Conclusion}

A l'issue des résultats de la présente étude, il est incontestable de reconnaître l'importance de l'approche participative CEP comme un canal de communication et de diffusion des technologies améliorées. Le processus a influé significativement sur le taux d'utilisation des technologies chez les producteurs ayant été directement impliqués dans les CEP et ceux n'ayant pas pris part. Un effet «tache d'huile» est perceptible pour la diffusion ou l'autodiffusion de paysan à paysan.

Les améliorations des taux d'adoption concernent l'utilisation des variétés améliorées, des fertilisants et la gestion des ravageurs des cultures qui sont des éléments déterminants pour l'augmentation de la production.

Dans le cas des variétés, la IT $90 \mathrm{~K}$ 372-1-2 est la plus utilisée, pourtant 16 génotypes bien adaptés aux conditions sahéliennes sont inscrits dans le catalogue national. Il y a lieu de poursuivre la promotion d'autres variétés améliorées avec le CEP et le renforcement des réseaux de production des semences pour fournir aux producteurs un choix varié.

L'utilisation des fertilisants concerne $1 / 5^{\text {ème }}$ des producteurs enquêtés. Le taux d'adoption est lié à la participation au processus CEP, mais aussi à l'accès au crédit. Il semble important de développer le crédit et aussi de renforcer les réseaux des boutiques d'intrants communautaires.

La pression des ravageurs des cultures est assez importante pour la plupart des producteurs et il est noté un faible taux d'adoption du biopesticide à base de grains de Neem en raison de la pénibilité de la technologie. Il est important de poursuivre les démonstrations à travers les CEP et aussi d'encourager le secteur privé à produire la solution prête à l'utilisation, à l'instar des pesticides.

\section{CONFLIT D'INTERETS}

Les auteurs attestent qu'il n'y a aucun conflit d'intérêts.

\section{CONTRIBUTIONS DES AUTEURS}

MMR a collecté, analysé les données et a rédigé le papier. IB a participé à l'analyse de données, la rédaction et la correction du manuscrit. RA a participé à l'analyse de données et la rédaction. LS a participé l'élaboration de l'outil de collecte des données et à la correction du manuscrit. LA a participé à la collecte et à l'analyse des données. BP a participé à la rédaction du manuscrit.

\section{REFERENCES}

Abebaw D, Haile MG. 2013. The impact of cooperatives on agricultural technology adoption: Empirical evidence from Ethiopia. Food. Policy, 38: 82-91. DOI: http://dx.doi.org/10.1016/j.foodpol.2012. 10.003

Adeoti R, Coulibaly O, Tamo M. 2002. Facteurs affectant l'adoption des nouvelles technologies du niébé Vigna unguiculata en Afrique de l'Ouest. Bull. Rech. Agron. Benin, 36: 18-26

Adesina AA, 1996. Factors affecting the adoption of fertilizers by rice farmers in Côte d'Ivoire. Nutr. Cycl. Agroecosys., 46: 29-39. DOI: 10.1007/BF00210222

Bationo A, Christianson CB, Mokwunye U. 1989. Soil fertility management of the pearl millet producing sandy soils of Sahelian West Africa: The Niger experience. In International Workshop on Soil, Crop, and Water Management Systems for Rainfed Agriculture in the Sudano-Sahelian Zone, Niamey (Niger), 11-16 Jan 1987. ICRISAT; 177-194

Bationo A, Mokwunye AU. 1991. Role of manures and crop residue in alleviating soil fertility constraints to crop production: With special reference to the Sahelian and Sudanian zones of West 
Africa. In alleviating soil fertility constraints to increased crop production in West Africa. Springer Netherlands; 217-225. DOI: 10.1007/978-94-0113224-4_19

Bellon MR, Becerril J, Adato M, Mindek D. 2006. Poor farmers' perceived benefits from different types of maize germplasm: The case of creolization in lowland tropical Mexico. World. Dev., 34(1): 113-129.

CIMMYT. 1993. The adoption of agricultural technology: A guide for survey design. Mexico, D.F.: CIMMYT. 88 pp, Retrouvé le 18/11/2016 sur le web à l'adresse:

https://www.google.fr/search?tbm=bks\& $\mathrm{hl}=\mathrm{fr} \& \mathrm{q}=$ The + adoption + of + agricultural + technology $\% 3 \mathrm{~A}+\mathrm{A}+$ guide + for+survey + design

Djinadou KA, Coulibaly O, Adégbidi AA. 2008b. Genre, Champ-école paysan et diffusion des technologies améliorées du niébé au Bénin. Bull. Rech. Agron. Benin, 60: 5159.

Djinadou KA, Coulibaly O, Agbo VA, Adégbidi AA. 2008a. Genre et accès aux facteurs de production pour l'adoption des extraits aqueux de Neem (Azadirachta indica) au Bénin. Les Cahiers de l'Economie Rurale, 6 : 69-80.

Dugje IY, Omoigui LO, Ekeleme F, Kamara AY, H Ajeigbe. 2009. Production $d u$ Niébé en Afrique de l'Ouest: Guide du paysan. IITA: Ibadan, Nigeria; 20p.

Dutordoir CD. 2006. Impact de pratiques de gestion de la fertilité sur les rendements en mil dans le Fakara (Niger). Travail de fin d'études présenté en vue de l'obtention du grade de bio-ingénieur. Retrouvé le 18/11/2016 sur le web à l'adresse: https://www.jircas.affrc.go.jp/ project/africa_dojo/Metadata/grad_resear $\mathrm{ch} / 03 . \mathrm{pdf}$
Egho EO. 2011. Management of major field in sect pests and yield of cowpea (Vigna unguiculata (L) walp) under calendar and monitored application of synthetic chemicals in Asaba, southern Nigeria. Am. J. Sci., 2(4): 592-602. http://www.scihub.org/AJSIR/PDF/2011 /4/AJSIR-2-4-592-602.pdf

FAO. 2011. Rapport de la mission d'évaluation indépendante du projet IARBIC. 70 p. Retrouvé le 19/11/2016 à l'adresse: $\quad$ http://www.fao.org/3/aas622f.pdf

Fischer E, Qaim M. 2012. Linking smallholders to markets:determinants and impacts of farmer collective action in Kenya. J. Dev. Stud. 40(6) : 12551268.

Hamadziripi A. 2008. Etude de cas : les associations villageoises d'épargne et de crédit du Niger le modèle Mata Masu Dubara de fourniture de services dans les zones reculés. Canada, Nova Scotia : COADY et Ford Foundation. 33p. Retrouvé le 18/11/2016 à l'adresse : http://www.coady2.stfx.ca/tinr oom/assets/file/ford/docs/translate/frenc h/Niger\%20(French).pdf

Ibro G, Bokar M. 2001. Transfert des nouvelles technologies dans le système de production des paysans au Niger. Etude de cas: adoption des variétés améliorées et de nouvelles techniques de production de la culture du niébé. Rapport soumis à l'IITA, projet PEDUNE, 17p. Retrouvé le 18/11/2016 à l'adresse http://www.fidafrique.net/ IMG/pdf/Technology_adoption_Pronaf_ Niger.pdf.

Jackai LEN, Inang EE, Nwobi P. 1992. The potential for controlling post flowering pests of cowpea, Vigna unguiculata Walp. using neem, Azadirachta indica A. Juss. Int. J. Pest. Manage, 38(1): 56- 
60. DOI: http://dx.doi.org/10.1080/ 09670879209371646

Jackai LEN, Oyediran I. 1991. The potential of neem, Azadirachta indica A. Juss., for the controlling post- flowering pests of cowpea, Vigna unguiculata Walp. I. The pod borer, Maruca vitrata. Insect. Sci. Appl. 12: 103-109. DOI: https://doi.org/10.1017/ S1742758400020580

Khonje M, Manda J, Alene AD, Kassie M. 2014. Analysis of Adoption and Impacts of Improved Maize Varieties in Eastern Zambia. World. Dev., 66: 696-706. DOI: http://dx.doi.org/10.1016/j.worlddev.201 4.09 .008

MA. 2012. Rapport d'évaluation préliminaire des récoltes 2012, et résultats de la campagne agricole 2012/2013, République du Niger, Ministère de l'agriculture. Direction des Statistiques 26p. Retrouvé le 18/11/2016 à l'adresse : http://www.reca-

niger.org/IMG/pdf/Evaluation_CampAgr i_fevrier_2013.pdf.

MA. 2016. Annuaire National de disponibilité en semences améliorées au Niger, Ministère de l'agriculture, $90 \mathrm{p}$. Retrouvé le 26/10/2016 à l'adresse : http://images.agri-profocus.nl/upload/ post/ANNUAIRE-DISPONIBILITESEMENCES_Niger_20161460556868.p df

MA. 2015. Résultats définitifs de la campagne agricole 2014; perspectives alimentaires 2014-2015: Ministère de l'agriculture. Direction des statistiques. 32p. Retrouvé sur le web le 04/11/2016 à l'adresse : http://www.recaniger.org/IMG/pdf/Resultats_definitifs_ Campagne_agricole_2014.pdf

MDA. 2008. Recensement général de l'agriculture et du cheptel, Résultats définitifs, vol VI, productivité des exploitations agricoles. 80p. Retrouvé sur le web le 28/11/2016 à l'adresse : http://www.instepp.umn.edu/sites/default /files/product/downloadable/Niger_2005 -07_Vol 6.pdf.

Miller M, Godfrey N, Levesque B. Stark E. 2009. The Case for Financial Literacy in Developing Countries: Promoting Access to Finance by Empowering Consumers, World Bank, DFID, OCDE et CGAP, Washington, DC. 13p. Retrouvé sur le web le 28/11/2016 à l'adresse:

https://www.globalbrigades.org/media/Fi nancial_Literacy.pdf.

Nkamleu GB, Coulibaly O. 2000. Le choix des méthodes de lutte contre les pestes dans les plantations de cacao et de café au Cameroun. Economie Rurale, 7: 5-11. DOI: $10.3406 /$ ecoru.2000.5208

Nkamleu GB, Adesina AA. 2000. Determinants of chemical input use in periurban lowland systems: bivariate probit analysis in Cameroon. Agr. Syst., 63: 111-121.

DOI:

http://dx.doi.org/10.1016/S0308521X(99)00074-8

Okoth J, Braun A, Delve R, Khamaala H, Khisa G, Julianus, T. 2006. The emergence of Farmer Field Schools Networks in Eastern Africa. Paper presented at the CAPRi. Research Workshop on Collective Action and Market Access for Smallholders 2-5 October 2006, Cali, Colombia. IFPRI, Washington, $10 \mathrm{p}$.

Osbahr H, Allan C. 2003. Indigenous knowledge of soil fertility management in southwest Niger. Geoderma, 111(3): 457-479. DOI: http:// dx.doi.org/10.1016/S00167061(02)00277-X

Oyewale RO, Bamaiyi LJ, Oparaeke AM, Adamu RS. 2014. Evaluation of four insecticide formulations for the 
management of insect pests of cowpea, J. Food Sci.Technol., 5(8): 180-188.

Rogers EM. 1995. The Diffusion of Innovations (4th edn). Free Press: New York.

Sale A, Folefack PD, Obwoyere GO, Lenah Wati N, Lendzemo WV, Wakponou A. 2014. Changements climatiques et déterminants d'adoption de la fumure organique dans la région semi-aride de Kibwezi au Kenya. Int. J. Biol. Chem. Sci., 8(2): 680-694. DOI: http://dx.doi.org/10.4314/ijbcs.v8i2.24
Saidou AK, Ajeigbe HA. BB Singh. 2011. Participatory Evaluation of Improved Cowpea Lines and Cropping Systems for Enhancing Food Security and Income Generation in Niger Republic, West Africa. American-Eurasian Journal of Agriculture and Environmental Science, 11: $55-61$.

Schultz TW. 1964. Transforming Traditional Agriculture. Yale University Press (Reprint), Arno Press: New Haven, New York; 206p. 